\title{
On-Orbit Maintenance Operations Strategy for the International Space Station - Concept and Implementation
}

\author{
Linda P. Patterson
}

\author{
DF53/Mechanisms and Maintenance Group, Systems Division, Mission Operations Directorate, \\ NASA/Johnson Space Center, Houston, TX 77058. (281)244-0077; linda.p.patterson1@jsc.nasa.gov
}

\begin{abstract}
The International Space Station (ISS) has an operational mission and profile that makes it a Logistics and Maintenance (L\&M) support challenge different from previous programs. It is permanently manned, assembled on orbit, and multi-national. With this technical and operational challenge, a unique approach is needed to support the hardware and crew. The key is the integration of on-orbit and ground analysis, supply, maintenance, and crew training into a coherent functional process that supports ISS goals and objectives. To integrate all the necessary aspects of hardware and personnel to support on-orbit maintenance, a myriad of products and processes must be created and coordinated, such that the right resources are in the right place at the right time to ensure continued ISS functionality. This paper will familiarize the audience with ISS On-Orbit Maintenance (OOM) concepts and capabilities for different maintenance tasks and discuss some of the logic behind their selection. It will also identify the operational maintenance support responsibility split between the U.S. and the various International Partners (IPs).
\end{abstract}

\section{ISS ON ORBIT MAINTENANCE OVERVIEW - INTRODUCTION}

An overview of the operational methods for performing Intravehicular (IVA) maintenance will be discussed. This capability will be required heavily during the assembly phase and for at least 10 years after assembly is complete. It is expected that the operational methodology for performing maintenance will evolve as more IVA experience is gained. However, an initial plan is required to allow for timeline planning, tools development, sparing, and crew training. Initial operational concepts will be outlined along with assumptions regarding how IVA operations will be conducted, both onboard and with ground Mission Control Center Houston (MCC-H) support. This will include: an operational maintenance philosophy; roles and responsibilities of the IPs; a summary of the different modes, types, levels, strategies and capabilities affecting OOM, and identification of tools to support OOM. While differences and similarities (standards) between IPs and the U.S. are discussed as they apply, the overall goal is universal, to minimize both the systems' downtime and the crew time to perform maintenance.

A key aspect of defining a process for successful OOM is that if this is not attainable, there will be no manned exploration beyond earth orbit. A means must be found to maintain the functionality of a complex vehicle long term, as a "long pole in the tent" of space exploration as it were. This is a whole new arena for space operations and this is the testing ground.

\section{MAINTENANCE PHILOSOPHY}

In order to better understand how the present philosophy of on orbit maintenance for ISS evolved, we need to look at some of the factors that make the ISS unique, relative to previous U.S. space vehicles.

1) The ISS never returns to the ground. Presently, mechanical malfunctions on the Space Shuttle are handled by applying temporary "fixes" which allow the Shuttle to continue to function for the short term safely both on orbit and during re-entry. Once on the ground, the vehicle is thoroughly inspected by an army of technicians and any defective systems or Line Replaceable Units (LRUs) are repaired or replaced. The result is a vehicle which is "like new" and ready for another launch. All ground "fixes" are considered to be permanent and provided in a controlled manner by an expert. Conversely, since the ISS never returns to the ground, all repairs must be made on orbit. Since the ISS is designed to operate for many years, all repairs must be designed to be permanent. (In space, long term temporary fixes are risky and over a period of time can actually consume more of the crew's time than permanent repairs). Also, all failures impact later missions or parts of missions so they must be allocated time and priority vs. science gathering. Here, the crew functions as both the operators and the maintainers of the systems.

2) The ISS vehicle will be assembled in orbit. The zero-g environment of space will cause special problems for crew members. Tools and loose Orbital Replacement Units (ORUs) will have to be tethered and small parts restrained so that they do not float away. Crew members will have to determine the best method of anchoring 
themselves while they attempt to remove and replace defective ORUs and parts. In the IVA environment, particles of debris caused by drilling and filing operations will have to be collected and disposed of so that they do not contaminate the crew's environment.

3) The lack of comprehensive end-to-end testing of the ISS' components carries a great deal of potential for subsequent problems. In an ideal world, all of the ISS' modules and trusses would be assembled on the ground and the mechanical and electrical interfaces between the various modules and trusses would be tested and any problems repaired. The vehicle would then be disassembled and the components carried into orbit by various launch vehicles. In the ISS program, this is not practical from a cost and schedule perspective. Many times the testing of the interface between two mating modules will be impossible, because while one module is being carried into space, the module with which it interfaces is still being assembled on the ground.

Given the potential for problems, the key to ISS success will be timely maintenance. Considering that the key criteria by which the ISS will be judged are the quality and quantity of science data produced, it is essential the science producing payloads are kept operating. To function nominally, payloads require ISS services such as electrical power, cooling, and command \& data handling $(C \& D H)$. You must have a C\&DH source path, to command power on, to provide heat/cooling to an ORU, especially as the ISS is a virtually $100 \% \mathrm{PC} / \mathrm{keyboard}$ controlled vehicle. To keep all of these services operating nominally, timely maintenance will be essential.

NASA and the Russian Space Agency (RSA) also have slightly different approaches to OOM of space stations:

- NASA's baseline approach for the ISS will be to R\&R defective ORUs in their entirety. In limited cases, where time considerations and the lack of a spare ORU do not permit replacing an ORU, repairs will be made to a part of an ORU (intermediate level). This approach is based on the idea that replacing ORUs requires less crew training and reduces the amount of crew time required to make repairs, thus increasing the amount of time to perform science. A tradeoff of up-weight and stowage space vs. complexity of repair and extensive crew skills training and fault diagnostics will become part of the equation in the decisions per ORU.

- The RSA approach for MIR is to repair ORUs in-situ (in place) on orbit. In some cases, where the particular ORU is a critical one, the temporarily repaired ORU will be replaced by a spare ORU when it becomes available via a Progress re-supply flight. In the past, RSA has had limited down-mass, requiring all ORUs to be maintained on-orbit (only a very small amount of mass can be returned on the Soyuz vehicle; the Progress vehicles burn up upon re-entry). Thus, generally, ground servicing has not been an option. The RSA method of maintenance was less of a choice than an only option.

The ISS has a choice and needs to make the best tradeoffs, based on lessons learned from past manned space and military maintenance and logistics, to ensure maximum cost savings, operational safety, and science benefit in the long term. Other factors influencing the maintenance strategy include: cultural uniqueness and respect for viable differences; methods and types of maintenance; complexity of tools/diagnostics; degrees of preplanning for maintenance (including on orbit sparing, prioritization or repair decisions, means of tracking tools on orbit, written procedures, etc.); and level of crew training required.

\section{Approaches: Totally Unified vs. Separate but Coordinated}

Logically there is always a best way to do a task. However, here, the task is not the sole driver in the decision. Two important factors are needed to put a country's OOM in the proper context: cultural views and historical success. These aspects will be applied to the current OOM philosophies to help understand why one unified philosophy was not the most workable or acceptable choice for all. Although the spaceflight experience of the ISS partners is varied, all parties agree that the safety of the crew and vehicle are paramount. Learning from each other, defining and negotiating Partner responsibilities, and implementing the best solution is not a simple process.

For example, the Russians do not need to plan as extensively for failures because they do not use as much new technology. The cultural aspect driving this is that new items are not used if an old, proven one will do the job. Much of the technology utilized on the Russian segment is based on proven Mir hardware. Stability is more important than change. Lack of change in design means spares are more plentiful and available and the expertise is available. Procedures for doing maintenance tasks are not as detailed because they expect the crew to be able to do tasks by using the extensive and in depth instruction they receive on the ground. The crew also has demonstrated more preference in dealing more off-the-cuff with problems rather than with extensive preplanning. The cultural aspect driving this is that Russians commit much more to memory than Americans. They also know their experts will be readily at hand as attrition is not a major problem in their industry. The Russians believe that if all is known the solution will come together at the time required and, so, there is little need for precise scheduling. Historically, their system, in their cultural context, has worked well.

Americans need to plan extensively for failures because new technology is widespread. The cultural aspect driving this is that 'newer is better'. The reliability of many items is unknown and reactions to failures must be heavily preplanned. Stability, especially in the new COTS tool and software upgrades world, can be perceived as a lack of 
progress. The U.S. culture favors the "new and improved, the more powerful". A failure is almost a challenge to demonstrate that you have planned well and that every contingency is considered. This also means there are few, if any, existing spares. Procedures for doing maintenance tasks are very detailed because the level of detail the crew is expected to recall is too little to perform a complex procedure from memory (i.e. low context, details orientation). The cultural aspect driving this is that Americans concentrate on knowing where to find information, not on memorizing every aspect of the information itself as the information often changes rapidly. Historically, this system has worked well for the U.S., although the implementation of so much new technology at one time has never before been attempted in space. The increased concentration on spares and procedures is the action needed to ensure safety in this cultural context. This information also needs to be documented in detail since individuals who possess the expertise may move on or be unavailable years later when actual failures might occur.

Partners should cooperate as much as possible, but we must also recognize our limitations. Unless a cooperative engineering and planning process is undertaken from the beginning, it is not practical to use one or the other partner's operational plan on all segments and in all cases. The cultural differences must also be evaluated to determine how to overcome them. Some are insurmountable, while others are not. It cannot be expected that there will be compromise on everything, or that compromise is always best. Nor can it be expected for one of us to adopt completely the practices of the other. The best way to ensure safety is to pick the 'better' way when it comes to critical issues, and experiment when it comes to lesser ones so that we may learn from each other. It will also be interesting to find, much the same as in the" nature vs. nurture" question of behavior, for space operations, which is "culture vs. experience" (i.e. what can be done either way, and what works best for all types of tasks).

The operational plan has to do with the way something is designed, and where the expertise for that hardware lies. The bottom line is that the U.S. and Russia each know their equipment, systems, and crew best, and each should be allowed, within the scope of the common agreements below, to make their own decisions on how the equipment will be operated and maintained. The following represents some pertinent key agreements (JSC 27099,1996):

- Emphasis is being placed on pre-written procedures and pre-positioned spares. Essentially, all critical and most common components will have a spare in a preplanned stowage location on orbit. The crew will have access to the collection of detailed procedures. While some procedures may be in multiple languages, the single common language onboard and for written documentation will be English.

- $\quad$ Preventive maintenance must be scheduled to reduce equipment failure and maintain redundancy levels.

- Corrective maintenance will be scheduled to restore a lost function. System malfunctions should be anticipated and procedures in place to regain function. Critical functions should be given priority in the crew timeline. Each system that is not considered critical will not inhibit vital operations between logistics launches.

- OOM operations should be assigned jointly between IPs and should be assigned to designated crew members, regardless of nationality and the country providing the module. However, for example, if a significant amount of OOM is to be done in a Russian module, it may be best to assign a Russian crewmember as one of the team.

- OOM operations will contain adequate safeguards to reduce hazards to the crew performing the task.

- The crew will be trained in skills to allow successful performance of OOM tasks.

The remainder of this paper will discuss the way maintenance practices and processes are set up as a "standard" for all participants. Variations will be noted as they apply to each Partner authoring its own procedures (often in both English and the native language) and in training the crew on their operations in their own mock-up facilities.

\section{International Partner (IP) Maintenance - Unique Roles \& Responsibilities}

The ISS requires cooperation from the various IPs in all areas, including maintenance. Considering the geographic, cultural, and language differences between IPs, this effort is very complex. IPs are responsible for the following activities for their respective components of Station hardware: integrating maintenance requirements, assigning priority, planning, crew training (at their mockup and IP facilities), and execution of maintenance procedures. In addition, each IP will be responsible for providing their own tool kits, although it is expected that tool sharing between IPs will occur. In some cases, one IP's spare parts may be delivered to orbit by another IP's launch vehicle. In the event of the loss of a component which is critical to crew safety, vehicle integrity, or mission success, and if the IPs agree, parts may be borrowed or "cannibalized" from one IP module and used in another. One function which may be shared by the IPs is training crewmembers in basic skills (such as soldering, splicing wires, repairing fluid lines, etc.). From a time perspective, it is more efficient to let one IP teach a particular skill to all crewmembers than it is for each IP to teach that skill to only their own crewmembers. Which IP has the most experience and best facilities for teaching a particular skill is to be the determining factor. Presently, an agreement is being negotiated between NASA and the IPs to determine an Integrated Maintenance Training Flow.

\section{MAINTENANCE CONCEPT: REPAIR STRATEGIES}


Any maintenance concept must define the rules and assumptions on which the repair strategies will be based. The following are details of the OOM modes, types and levels, as well as the inclusion of imagery and tool fit check requirements for the hardware.

1) Modes of On Orbit Maintenance: OOM is performed to sustain an acceptable level of system operability and to ensure station survival, crew safety, mission success, and payload operations support. There are three modes:

- IVA (Intravehicular Activity) - Performed inside the vehicle

- EVA (Extravehicular Activity) - Performed outside the vehicle, using special EVA tools, restraints and aids (Crew and Equipment Translation Aid (CETA) cart)

- EVR (Extravehicular Robotics) - Using the Space Station Remote Manipulator System (SSRMS) alone or in conjunction with EVA to perform external maintenance. Using the SSRMS alone to perform external maintenance is the preferred method, since this minimizes the crewmembers' exposure to the hazards of the space environment and does not limit the operations to the 6 hours of EVA suit time. When performed in conjunction with EVA, the SSRMS may be used to move the work to the crewmember, or vice versa.

Many OOM procedures will utilize multiple modes. For example:

- For a failed external video camera luminaire unit, the SSRMS is used to position EVA crewmember in the vicinity of the luminaire assembly. The crewmember removes the assembly and takes it inside. IVA, the bulb is removed and replaced (at the Maintenance Work Area (MWA)). This entire process is then reversed to put the luminaire assembly back in its original location.

- A possible alternate method for some ORUs, such as an external Multiplexer/Demultiplexer (MDM), could be that a duplicate of the failed unit is "built" at the MWA and then the new replacement is taken EVA/EVR. When the R\&R is complete, the failed unit is returned IVA where it may be repaired and now function as a future spare (or returned to the ground for deeper analysis). This significantly reduces the EVA time and external operations but can be used in only a limited number of scenarios.

Table 1 clarifies that IVA will take up the lion's share of crew time applied to maintenance. EVA is naturally limiting because of consumables and crew fatigue issues. The primary mode of maintenance for external hardware is by EVR, which is the use of the dexterous robot (telerobot). EVR is performed where there is telerobotic compatibility and availability. EVR has advantages but is costly in design. The backup for EVR maintenance is EVA. Cooperative EVA/EVR maintenance includes using robotic capability to translate hardware that has too much mass for crewmembers to handle or to position the crewmember at specific worksites. (SSP 50520,2000)

TABLE 1. Estimates for ISS Crew Time for Maintenance.

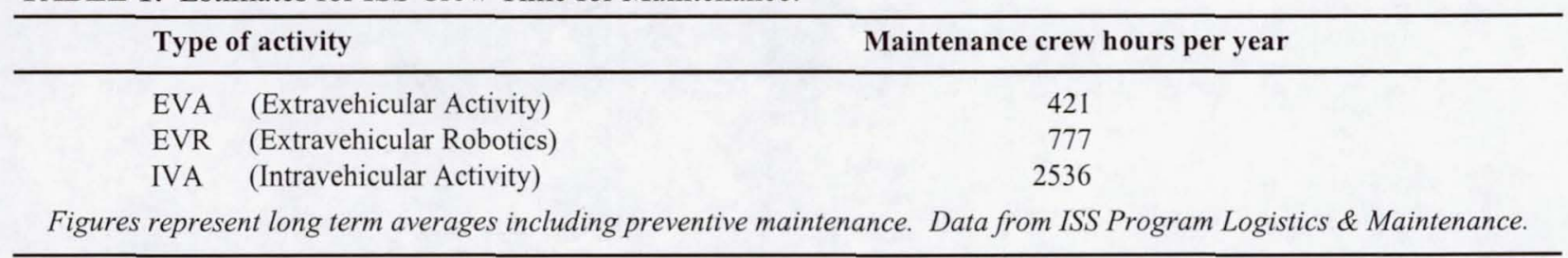

2) Types of On Orbit Maintenance: The following categories of maintenance are based on either the urgency of the maintenance, the time frame, or the place the maintenance will be carried out.

- Preventive - To keep item(s) in a specified condition by performing systematic inspection, detection, cleaning, repair and/or replacement of parts at pre-planned, specified intervals. This is a move away from the past philosophy of "run it till it breaks" or "if it ain't broke, don't fix it" for most all hardware. This is to reduce equipment failure and so prevent corrective maintenance.

- Corrective - To restore an item to its original condition. This is usually pre-planned and the crew has a replacement and current specific skills training on the task.

- In-Situ - Repairs performed at the hardware site.

- Contingency - Maintenance performed to restore a function which is vital to crew safety or vehicle integrity. This is usually an unanticipated event and may require immediate action and a reprioritization of crew plans for other work/maintenance.

There may be overlaps between different types of maintenance. If corrective maintenance is being performed on an ORU and the ORU cannot be removed for servicing, then the maintenance is also in-situ maintenance.

3) Levels of On Orbit Maintenance: The ISS uses three levels: organizational, intermediate and depot. Each succeeding level requires a higher level of skill and more complex tools/diagnostic equipment. Details in Table 2.

An ISS MDM can be used to illustrate the various levels of OOM: Organizational if one removes a defective MDM and replaces it with a spare; Intermediate if one removes a defective MDM, carries it to the MWA, opens the MDM, removes a defective circuit card and replaces it with a spare card; Depot if the defective circuit card is then carried to the ground where a failed integrated circuit chip on the card is replaced. 
TABLE 2. Maintenance Levels.

\begin{tabular}{|c|c|c|c|}
\hline Level (Location) & Skills & Equipment required & Example \\
\hline $\begin{array}{l}\text { Organizational - } \\
\text { (performed on } \\
\text { orbit) }\end{array}$ & $\begin{array}{l}\text { Minimal } \\
\text { mainten- } \\
\text { ance skills }\end{array}$ & $\begin{array}{l}\text { Standard hand tools, some } \\
\text { diagnostic equipment }\end{array}$ & $\begin{array}{ll}\text { - } & \text { Visual inspections } \\
\text { - } \& R \text { of some ORUs } \\
\text { - } & \text { Periodic cleaning/servicing of equipment } \\
\quad \text { External adjust/align ORUs }\end{array}$ \\
\hline $\begin{array}{l}\text { Intermediate } \\
\text { or I-Level - } \\
\text { (performed IVA } \\
\text { on orbit or on } \\
\text { ground) }\end{array}$ & $\begin{array}{l}\text { Higher } \\
\text { level of } \\
\text { skill(s) }\end{array}$ & $\begin{array}{l}\text { More support/diagnostic } \\
\text { equipment than } \\
\text { organizational } \\
\text { maintenance }\end{array}$ & $\begin{array}{l}\text { - Diagnosis and isolation of malfunctions; adjustment } \\
\text { and alignment of modules readily accomplished with } \\
\text { test, measurement, and diagnostic equipment on-orbit. } \\
\text { Repair by replacing defective modular components and } \\
\text { piece parts, such as transistors, transformers, etc., when } \\
\text { replacement will recover functionality without } \\
\text { extensive post-maintenance testing. } \\
\text { Repair of cables to include wire/optical splicing, pin } \\
\text { straightening, replacement of worn insulation, etc. }\end{array}$ \\
\hline $\begin{array}{l}\text { Depot - } \\
\text { (normally } \\
\text { performed on the } \\
\text { ground) }\end{array}$ & & $\begin{array}{l}\text { Specialized equipment not } \\
\text { available on orbit, } \\
\text { extensive collections of } \\
\text { spare parts, complex } \\
\text { diagnostic equipment }\end{array}$ & $\begin{array}{l}\text { - Complete overhauling/rebuilding of equipment (such } \\
\text { as failed circuit cards). Complex calibrations of } \\
\text { equipment. }\end{array}$ \\
\hline
\end{tabular}

The I-Level is a newer level, for use in the U.S. space program, and considered for ISS due to up-mass and stowage limitations. However, replacing a part of a whole is limited since many ORUs may be sealed or beyond the scope of troubleshooting beyond the black box level for replacements of parts. The potential hazard to crew or equipment is also considered. Many items may not be simply "plug-in" and the desire is to limit the level of skills required for a successful change-out and to reserve the crew time mainly for science, rather than increasing repair skills.

4) Imagery and Fit Checks: All partners have been made aware of the rationale and value vs. cost of incorporating both hardware imagery and fit checks into their assembly. Central electronic database storage and access capabilities have been made available for partners. Never more than in this program has imagery (still photographs and video) of the complete interior and exterior of the elements been required. There is no "hangar queen" budgeted by any partner so once the hardware is launched, there is no way to investigate issues or ensure correct trainer outfitting without imagery cross-checking. Similarly, as many procedures will not be created until the item has failed, the imagery produced by the hardware providers (during the manufacture, test, build and closeout) and imagery taken during ground installation and fit check opportunities, will be a key verification source. Every crew has stated the desire to have included in the procedures numerous photos for clarity, recall, and increased speed of review and task completion. Fit checks are opportunities to test fit the hand tools on the hardware during element build on the ground. This is an invaluable aspect of maintenance procedure verification.

\section{ON ORBIT MAINTENANCE STRATEGY}

The ISS On-Orbit Maintenance strategy involves both hardware and support elements. Hardware elements include tools and spare parts. Support elements, concerned with the ground MCC function of preparation for real time ops, include procedures creation and crew training.

\section{Hardware Elements}

U. S. OOM hardware elements consist of U. S. tools, incorporating hand tools and diagnostic tools, as well as spare parts. This stowage vs. up-mass trade-off provisioning is key to any successful plan for OOM.

Hand tools can be subdivided into EVA hand tools and IVA hand tools. The main differences between the two types of tools is that the EVA tools have special provisions for tethering and use by (EVA) gloved hands. The IVA hand tools are basically the same type of common tools found in most garages or machine shops. The goal is to define a set of common tools of maximum use to all users so that stowage volume and weight are reduced. The following is a list of typical types of IVA hand tools stowed in the ISS (Note that this is not a complete list): Ratchets (powered/unpowered, torque, adapters, universal joints, breaker bars, extensions), Sockets (regular/deep, hex head), Screw drivers (common-tip, Phillips head, jeweler's), Wrenches (open/box end, L-shaped, hex head), Pliers, Metal working tools (hacksaw, bone saw, chisels, punches, files), and Hammer (deadblow, ball peen). 
There is more than one set of IVA hand tools on the ISS. The following sets will also be on the ISS:

- IP hand tools - Each IP will have a set for their module(s) (RSA, ESA, NASDA). For common tools, sharing of the U.S. common set is expected by all partners and payloads.

- Payload tools - Each payload will be expected to provide any special tools needed to maintain their payload.

- Special tools - For certain ORUs, special tools will be required to remove and replace the ORU. In most cases, these special tools will be packed with the replacement ORU.

- Specialty tool kits such as: Electrical repair tools, Fiber Optics repair kit, Fluid Line Repair Kit, Hose \& cable kit (similar to shuttle kit), Tap \& die set.

The NASA IVA hand tool set for the ISS is unique in that it contains both English and metric tools. The U.S. Hardware providers are mostly from the U.S. and so the hardware was manufactured to English standards. Most of the IP tool sets will contain only metric tools. For any hardware provided by U.S. companies, not in metric, they will use the common tools. This tool set contains most of the tools which were in the shuttle tool set and in addition, a large number of new tools. Within a storage bag, the tools are grouped into "kits" of like tools.

Lessons learned from the first ISS missions have indicated the need for more redundancy in the frequently used tools. This allows not only for simultaneous maintenance in multiple locations (requiring the same tool) but also cuts down time for the overhead of getting the tools out and returning them to the folded pouches each time. It also reduces the risk of not being able to perform a task due to tool loss or damage. To reduce access time to the tools, a set of tool drawers is being created to replace some of the bags for the frequently needed tools. It is also expected there will need to be an inventory pre-mission of key tools required for any upcoming mission's maintenance to ensure the tools are available and in working order. Lessons learned from the Mir station have indicated either that a tracking system of the tools onboard must exist or key tools must be flown with the ORU or both. A barcode tracking system is planned for the ISS but an inventory check before each support vehicle launch is also planned so anything required for upcoming maintenance that is missing can be brought up on that next launch.

Diagnostic tools are primarily used to perform fault isolation when the exact fault cannot be identified to an ORU or there is a gain to be realized if I-Level sub-ORU maintenance is to be performed. Generally, the procedure is to remove a defective ORU, carry it to the MWA, open it and use the diagnostic equipment to pinpoint which electrical component of the ORU has failed and in what manner. Once the defective ORU component is replaced, the diagnostic equipment is again used to determine if the repair was successful. After this, the ORU is closed and returned to its original location. If the ORU cannot be removed from its installed location, the diagnostic tools can be carried to the ORU and the maintenance performed in-situ (this can also involve the analysis of locations of faults in line runs for the electrical or data systems). A description of some diagnostic tools follows:

- MWA and Containment System: A portable, foldable work table (36 " $\mathrm{x} 25$ ") which can stowed. It clamps to tracks on either side of a pressurized element "wall" face. It can be used to restrain ORUs while maintenance is being performed. Slots allow the restraint of ORUs with bungee cords. The Containment System is a clear plastic enclosure designed to contain debris created by OOM operations such as cutting, drilling, or soldering.

- Scopemeter: A commercially available combination of multimeter and oscilloscope.

- Logic Analyzer: Consists of a Portable Computer System (PCS), a Portable Computer Memory Card International Adapter (PCMCIA) and LabVIEW software. The PCMCIA card has numerous probes connected to it which allow the PCS to monitor several different points in a circuit, or several circuits, simultaneously.

- Function/Sweep Generator: Generates standard waveforms to diagnose circuits and perform fault isolation.

- Pin Kit: Material to create new connectors or work on damaged ones (ex. fabricate custom jumper/test cables).

Spare Parts - As can be seen from the ISS tools list, the crew has a great deal of capability to diagnose and repair failed ISS ORUs and ORU components. Although some repairs can be performed by simply tightening a bolt or adjusting a seal, most repairs require a spare ORU or spare parts for an ORU. Successful spares provisioning must include (ground and onboard): procurement; storage; inventory; transportation; and tracking of ORU failure modes and lifetime. For the ISS program, a quantity of selected spares were purchased before any element was launched. Due to financial considerations, it is impossible to provide spares for every ORU. The following factors were considered when determining which items to spare:

- The criticality of the ORU to crew safety, vehicle integrity, or mission success

- Mean Time Between Failures (MTBF) - (based on tests of a quantity of the ORU; the average length of time the ORU operates without failing.)

- The quantity of that particular type of ORU on the ISS

- The availability of operational "work-arounds" if the ORU fails

- The availability of launch vehicle volume and weight allowance to carry the ORU to orbit, on demand

Spares are categorized as one of the following:

1) Preplanned - a spare part purchased, based on the above criteria, and scheduled to fly on a future mission.

2) Prepositioned - a spare, already onboard the ISS and available for use.

3) Backlogged - if an ORU fails and a spare cannot be acquired in a timely manner, the required OOM is postponed.

4) "Borrowed" or "cannibalized" - if an ORU performing a critical function fails, and another ORU of its type is available on orbit, and is performing a function deemed less critical than the first, the second ORU may be 
"borrowed" to perform the more critical function. When a spare becomes available, it is placed in the position of the second ORU; the borrowed ORU, performing the more critical function, is not replaced.

\section{Support Elements}

To complete the picture of maintenance integration, the ground support element must be introduced. The presence of hardware alone is not sufficient to enable the concept of maintenance to succeed. There must be a skilled crew to perform the event and a skilled ground support staff to author the procedures and train the crew to do the tasks. These ground experts become involved in the process very early, during requirements and design phases, to ensure the final product is maintainable (i.e. ORU accessibility, fault identification to a sufficient level).

The Mission Control Center Houston (MCC-H), operated by the Mission Operations Directorate (MOD), is responsible for near real-time level planning and on-orbit execution. The MCC-H coordinates across Partner control centers, where the operations experts for each system are integrated into a team, though they may not be colocated. Audio, visual, and data links connect the experts globally in order to maximize data sharing and integration. MCC-H flight controllers are responsible for identifying anomalies and determining hardware failures. They work with Partner control centers to isolate anomalies across Partner systems. The MCC-H is responsible for scheduling and execution of OOM tasks, for assessing real-time anomalies, and determining whether to use an onorbit spare to restore system operability. The Operations Support Office (OSO) is the position in the MCC-H tasked with OOM. The OSO tasks include but are not limited to the following activities in support of OOM and logistics:

- Assisting in Failure Detection, Isolation, and Recovery (FDIR) of anomalous systems by working with systems disciplines and Partner control centers to isolate anomalies across Partner systems.

- Tracking and reporting of ORU failures and recording the execution of maintenance tasks

- Allocating Maintenance Backlog into a Prioritization

- Determination of the availability of spare ORUs

- Generation of maintenance procedures (both pre-mission and real-time)

- Scheduling and execution of maintenance tasks

- Updating of maintenance databases to reflect current vehicle status

- Training the crew on skills

- Defining the level of fidelity of maintenance training facilities

- Defining the level of PC-based tools and applications (this involves requirement definition and often the creation or refinement in-house of the tool)

The OSO interfaces with the ISS Flight Director and other flight team personnel to assist with Critical Failure Response and Anomaly Resolutions. This includes assessing anomaly/failure situations and providing corrective action options. They also work with the ISS Program Office Logistics and Maintenance (L\&M) personnel on developing options involving additional source data, parts/tools availability, contingency maintenance capabilities, ORU manifesting, preventive maintenance, and backlog management. The OSO works with the L\&M personnel to determine whether to attempt a contingency workaround or to defer maintenance until the spare can be manifested.

It is not always possible or feasible to replace and restore functionality immediately upon failure. At any time during the life of the ISS, there are expected to be numerous ORUs which have failed or degraded, forming a backlog of maintenance tasks. Some of these failures may be serious, while others may have less impact. With the limited resources available (particularly crew time) a hierarchy was developed which allows prioritizing of these repairs to make the best use of the available resources. The prioritization hierarchy is as follows (SSP 50520, 2000) Hardware which supports systems and functions:

Priority 1 (Station/Crew Survival): Required to maintain a stable attitude adequate to conduct proximity operations and emergency separations and departure and prevent the crew from being exposed to life threatening conditions. Priority 2 (Assembly/Element Survival and Revival): Required to mate a delivered launch package to an existing stage to perform activation and checkout, maintain element survival and/or the ability to revive a lost element. Priority 3 (Re-supply and Crew Rotation): Required to perform ISS Re-supply and Crew Rotation. Priority 4 (Crew Habitation): Required to maintain acceptable conditions to support long duration crew operations. Priority 5 (Payloads/Research): Required to support ISS payload operations and utilization. Priority 6 (Non-Essential): Not essential to ISS survival, habitability, and utilization.

This supports the maintenance planning process by showing the relative impact of failures on vehicle operability and payload utilities. For U.S. core system hardware, the OSO is responsible for assigning a maintenance priority to each failure and for providing a measure of the relative impact of the failure on the onboard systems operations, crew safety, vehicle operability, and payloads. The OSO is responsible for entering the maintenance prioritization for each failed or degraded U.S. hardware item. International Partners are to prioritize failures of their hardware using the same prioritization criteria. The maintenance priorities are recommendations used for maintenance planning. Maintenance priorities can be updated by management decisions. 
Maintenance Procedures - Procedure Elements are a sequenced set of steps that describe how to remove, replace, repair, inspect, calibrate, or adjust an ORU or ORU components. Most ISS core maintenance procedures contain the following standardized eight basic elements: 1) List of required tools/spare parts; 2) Safing steps such as removing power or pressure from the site so as to ensure crew safety in R\&R; 3) Access steps taken to get access to a failed ORU which may require removing panels or rotating racks; 4) Remove steps; 5) Replace/Install steps; 6) Check-out steps to ensure the new part works in its new location; 7) Close-out steps reinstalls any access panels removed; 8) Post-maintenance stowing of tools and the defective ORU and maintenance record keeping.

The standardization is essential to ease training and improve clarity especially with respect to an international crew. The IPs have agreed to adhere to a standard set of guidelines. For all maintenance related procedures, for all IPs and payloads, the OSO will review the procedure before its use as a "sanity" check to ensure standards have been followed and the task steps would be clear to the crew (both by order and functionality).

Maintenance Procedures - Procedure Generation is an on-going effort for as many probable failures as possible. However, it is obvious that due to the sheer volume of possible failure permutations, it would be impractical to create all procedures ahead of time. There will be failures for which there are no pre-defined maintenance procedures. When this occurs, the necessary procedures will have to be written "real-time" as required. The unique aspect here is that this vehicle does not have to be concerned with re-entry in a few days so there is time to deal with long term support, especially if there is no spare available. There may be months to define the procedures before the replacement hardware is made available on orbit. The real time procedures can be sent up with the upcoming crew or can be uplinked from the ground. Those procedures for critical hardware, or on orbit spared hardware, or "planned to be flown this mission" hardware, will be created and verified before launch.

Maintenance Procedures - Procedure Location for some basic predefined subset is onboard stored on a CD ROM or as part of the System Operation Data File (SODF) and accessed by a PCS. Additional procedures are uplinked from the ground as required. As a minimum, the following procedure types are stored onboard: Routine/Preventative (performed on a relatively frequent basis), and Emergency (immediate access by crew required).

Maintenance Training - As mentioned before, crew training in maintenance techniques enhances the ability to make successful repairs. All crewmembers receive basic and advanced training in OOM techniques. Selected members receive additional, increment-specific training. As time does not permit training the crew on all possible OOM procedures, the emphasis is on teaching basic skills. However, some routine and critical maintenance procedures are covered in training. Only the most critical and the most common procedures are taught on the ground. IPs and payload providers are responsible for providing crew training for their specific modules/equipment. This training takes place at their respective facilities (based on mockup fidelity). NASA and RSA may coordinate the teaching of basic maintenance skills (i.e., NASA might teach gluing skills; RSA might teach soldering skills, etc.).

\section{CONCLUSION}

The key to successful maintenance of the ISS is the integration of on-orbit and ground analysis and planning, supply, maintenance procedures, and crew training into a coherent functional process that supports the goals, objectives, and the mission of the ISS from the perspective of all partners in the ISS venture. To properly integrate all the necessary aspects of hardware and personnel to support on-orbit maintenance, a myriad of products and processes must be created and coordinated, such that the right resources are in the right place at the right time to ensure continued ISS functionality. There is an ongoing challenge for the U.S. and the IPs to overcome the cultural differences inherent with each partner. The final outcome will be a mixture of styles that is more workable than any single approach would have been. Much effort has been spent seeking understanding and agreement. The productive operation of the ISS throughout its life will be the best testimony to the success of this effort.

\section{ACKNOWLEDGMENTS}

The 16 countries participating in the ISS Program are: USA, Russia, Canada, Japan, Belgium*, Denmark*, France*, Germany*, Italy*, Netherlands*, Norway*, Spain*, Sweden*, Switzerland*, U.K.*, and Brazil.

* represent members of the European Space Agency.

\section{REFERENCES}

SSP 50520, International Space Station Logistics \& Maintenance Operational Support, Baseline Draft, April 2000.

TD9702A, International Space Station Familiarization (ISS FAM C 21109 training manual), Mission Operations Directorate, Space Flight Training Division, Section 12: On Orbit Maintenance Overview, OSO subject matter experts, July 1998.

JSC 27099, Space Station Flight Crew Operations Concept, Revision 2, December 1996. 


\title{
On-Orbit Maintenance Operations Strategy for the International Space Station -Concept and Implementation
}

\author{
Linda P. Patterson \\ DF53/Mechanisms and Maintenance Group, Systems Division, Mission Operations Directorate, \\ NASA/Johnson Space Center, Houston, TX 77058. (281)244-0077; linda.p.patterson1@jsc.nasa.gov
}

\begin{abstract}
The International Space Station (ISS) has an operational mission and profile that makes it a Logistics and Maintenance support challenge different from any previous program. It is permanently manned, assembled on orbit, and multinational. It is an on-orbit station that must be supported by a single integrated crew 24 hours a day, 7 days a week, and 365 days a year. In light of this technical and operational challenge, a unique approach is needed to support the hardware and crew. The purpose of the supportability effort is to not only manage logistics but also plan for, and conduct, maintenance operations to ensure that the on-orbit vehicle, and its associated systems, support safe, successful operation and science utilization.
\end{abstract}

The key is the integration of on-orbit and ground analysis, supply, maintenance, and crew training into a coherent functional process that supports the goals, objectives, and the mission of the ISS. To properly integrate all the necessary aspects of hardware and personnel to support on-orbit maintenance, a myriad of products and processes must be created and coordinated, such that the right resources are in the right place at the right time to ensure continued ISS functionality.

Operations support activities and processes are embedded in the full range of a program, from hardware development through mission operations. Readiness for maintenance operations involves ensuring first that the vehicle is maintainable. This includes paralleling operations planning for support during the design, manufacture, assembly test and closeout timeframe. The real time phase includes both ground and on-orbit operations beginning in the on-orbit assembly timeframe and continuing for the life of the program.

Space Shuttle and Mir supportability concepts were reviewed as a basis for building the concept for support of ISS. However, although experience from these programs serves as a starting point, the support concept for ISS has, by necessity, evolved. Continuous on-orbit operation requires that all maintenance be performed by the crew, in contrast to Shuttle where most maintenance is performed by ground support personnel between missions. More than any previous program, ground and on-orbit operations must be synchronized. Maintenance planning, repair strategies, etc., must be coordinated long before the task is required (during the design and build of the hardware). This coordination and synchronization is the key to providing essential resources and skills for successful maintenance in a timely manner to maximize ISS utilization. Effective integration of hardware and crew support requires that the ground infrastructure effectively supports the on-orbit vehicle and crew. Improved communications between ISS and the Mission Control Center allows closer coordination between support specialists on the ground and the on-orbit crew than was possible with Mir.

With the initiation of on-orbit operations of the ISS, emphasis is shifting from concept and strategy development to implementation. This paper will familiarize the audience with ISS On-Orbit Maintenance concepts and capabilities (philosophy, methods, types, levels, priorities, etc.) for different maintenance tasks and discuss some of the logic behind their selection. It will also identify the operational maintenance support responsibility split between the U.S. and the various International Partners. 